Revue des patrimoines

$36 \mid 2018$

Les archives photographiques de presse, pratiques comparées et enjeux méthodologiques

\title{
Exposer une revue : l'exemple de Réalités (1946-1964). Maison européenne de la photographie (Paris), 16 janvier - 30 mars 2008
}

Exhibiting a magazine, Réalités (1946-1964), on show at the Maison européenne de la photographie (Paris) from 16 January to 30 March 2008

\section{Anne de Mondenard}

\section{(2) OpenEdition}

Journals

Édition électronique

URL : http://journals.openedition.org/insitu/18482

DOI : $10.4000 /$ insitu. 18482

ISSN : $1630-7305$

Éditeur

Ministère de la culture

Référence électronique

Anne de Mondenard, "Exposer une revue : l'exemple de Réalités (1946-1964). Maison européenne de la photographie (Paris), 16 janvier - 30 mars 2008 », In Situ [En ligne], 36 | 2018, mis en ligne le 18 octobre 2018, consulté le 03 mai 2019. URL : http://journals.openedition.org/insitu/18482 ; DOI : 10.4000/insitu. 18482

Ce document a été généré automatiquement le 3 mai 2019.

In Situ Revues des patrimoines est mis à disposition selon les termes de la licence Creative Commons Attribution - Pas d'Utilisation Commerciale - Pas de Modification 4.0 International. 


\title{
Exposer une revue : l'exemple de Réalités (1946-1964). Maison européenne de la photographie (Paris), 16 janvier - 30 mars 2008
}

\author{
Exhibiting a magazine, Réalités (1946-1964), on show at the Maison européenne \\ de la photographie (Paris) from 16 January to 30 March 2008
}

Anne de Mondenard

1 Comment rendre compte, à travers une exposition muséale, de la place de la photographie dans une revue illustrée ? Exposer des tirages des images publiées en les contextualisant ou présenter la revue elle-même, à travers un choix de couvertures et de doubles-pages ? Le propos de cet article est de retracer les circonstances et les enjeux qui ont présidé à la présentation de l'exposition «Réalités, un mensuel illustré français (1946-1978) » à la Maison européenne de la photographie, en 2008¹.

\section{Pourquoi Réalités ?}

Quelques mots sur ce mensuel créé en 1946 sur le modèle du magazine américain Fortune, dont les sujets haut de gamme destinés à un public cultivé lui assurèrent un grand succès, avant de disparaître en 1978, au point d'être totalement oublié en 2008, quand nous lui avons consacré un ouvrage et une exposition². Son ancien rédacteur en chef, Alfred Max (1913-1990), rappelait ainsi en 1988 la cible visée : «Tous ceux curieux de connaître et ambitieux de réussir ». Il en résumait la forme :

un papier de poids, une couverture attrayante, les procédés d'impression les plus modernes, le recours fréquent à la couleur, un luxe de bon aloi » sans oublier le fond : " une quinzaine d'articles nerveux, substantiels, novateurs, couvrant tous les centres d'intérêt de nos lecteurs potentiels, traités en équipe par des chercheurs et des auteurs de talent que je formerais à se couler dans le moule de la revue, au lieu de briller et de se faire valoir individuellement comme c'était la règle pour la 
plupart des publications françaises. L'ensemble serait complété par quelques rubriques spécialisées signées de noms connus (Louis Armand, Raymond Aron) sans exclure l'humour (la réalité dépasse la fiction) ${ }^{3}$. Réalités devient un des titres les plus novateurs et les plus influents des années 1950-1960. Son audience le place parmi les journaux les plus lus et les plus regardés en France, avec en moyenne 160000 exemplaires vendus, essentiellement sur abonnement, et plus d'un million de lecteurs. accompagne l'expansion économique des trois décennies de l'après-guerre - les Trente Glorieuses - et raconte la société de consommation naissante. Elle ambitionne d'être «un observatoire du monde » en inventant un modèle journalistique : traiter de l'actualité à un rythme mensuel. Pour raconter la Chine de Mao, l'Espagne de Franco, la guerre d'Algérie, mais aussi le Touareg ou le pêcheur de l'île de Sein, le mensuel envoie des reporters et des photographes aux quatre coins de la planète, plusieurs mois s'il le faut, à une époque où quasiment personne ne voyage et où la télévision est balbutiante.

Les images, majoritairement en noir et blanc au début, se déploient progressivement en couleur $^{4}$. Les petits formats laissent aussi la place à de plus en plus de pleines pages. Elles sont souvent réalisées par des auteurs salariés de la revue. Les plus réputés sont Édouard Boubat (1923-1999) et Jean-Philippe Charbonnier (1921-2004). L'équipe fixe comprend encore Michel Desjardins (né en 1933) et Jean-Louis Swiners (né en 1935) ainsi que des collaborateurs réguliers comme Frank Horvat (né en 1928), Gilles Ehrmann (1928-2005) ou Jean-Pierre Sudre (1921-1997). Les plus grands photographes internationaux des années 1950 et 1960 sont également publiés dans Réalités : Brassaï, Henri Cartier-Bresson, Cecil Beaton, Ernst Haas, Richard Avedon, Irving Penn, etc.

6 Réalités est aussi à l'origine d'un groupe de presse et d'édition. En plus de Réalités, la Société d'études et de publications économiques (SEPE) lance en 1947 la revue Réalités littéraires. En partenariat avec Hachette, la SEPE publie des livres d'art comme Le XVIII ${ }^{e}$ siècle français (1956) et Le XIX siècle français (1957), sous la direction de Stéphane Faniel, avant de lancer avec Les Merveilles du monde (1957) sa collection la plus populaire. Suivront notamment, après ce best-seller, Les Merveilles de la France (1961-1962) et Les Merveilles de l'Europe (1963). Le groupe lance également sept revues spécialisées. La première à voir le jour, en 1952, est Connaissance des arts. C'est aussi le seul titre du groupe qui subsiste. En 1953, le groupe lance aussi la revue Entreprise, rachète le magazine pour adolescents Benjamin, en 1958, qui est rebaptisé Top ${ }^{5}$. Au début des années 1960, la SEPE cède la totalité de son capital à Hachette. Cette cession n'a d'abord aucune incidence. Mais le groupe est démantelé en 1974. Cette date coïncide avec le départ des trois hommes qui animent Réalités: le rédacteur en chef, Alfred Max et les deux directeurs fondateurs, Didier Rémon (1922-2018) et Humbert Frèrejean (1914-2001). Réalités disparaît en 1978.

7 Pourquoi exposer Réalités trente ans après sa disparition? Le photographe Jean-Philippe Charbonnier, rencontré à la fin des années 1980 à la galerie Agathe Gaillard, nous a incitée à nous intéresser à cette revue. Charbonnier était un personnage haut en couleur et très volubile mais qui devenait très sérieux quand il évoquait la revue pour laquelle il a travaillé pendant plus de vingt ans. Il en avait été le premier photographe attitré, en 1950, et se désolait de voir progressivement disparaître des témoins qui pourraient rendre compte de l'histoire d'une revue qui n'avait pas misé sur la photographie lors de son lancement mais qui l'avait largement accueillie et mise en valeur dans ses pages. 
D'abord intriguée par cette revue oubliée, nous avons commencé à dépouiller les premiers numéros au début des années 1990, en même temps que d'autres revues des années 1930 à 1960, pour y collecter des informations, sans formuler de projet précis. Quand l'éditeur Larousse a sollicité de jeunes chercheurs pour la première édition de son Dictionnaire de la photographie (1994), nous avons proposé, en plus de notices sur des photographes d'après-guerre, des textes concernant la presse illustrée et notamment l'hebdomadaire $V u$ et le mensuel Réalités. Par la suite, accaparée par d'autres recherches sur les débuts de la photographie, nous n'avons pas poursuivi nos investigations sur les journaux illustrés.

En 1999, quand le photographe Édouard Boubat est décédé, Jean-Philippe Charbonnier était en colère de voir partir un témoin essentiel de l'histoire de Réalités sans qu'aucun travail d'ampleur n'ait été entrepris. Puis Jean-Philippe Charbonnier est mort à son tour, en 2004. Il ne fallait plus attendre. En 2006, nous avons proposé une exposition sur la revue Réalités à Jean-Luc Monterosso, directeur de la Maison européenne de la photographie (MEP). Il était en train de programmer une exposition rétrospective sur l'œuvre d'Édouard Boubat en 2008 et a immédiatement adhéré au projet. Il connaissait la revue. En 1983, dans l'introduction au catalogue de la rétrospective Jean-Philippe Charbonnier au musée d'Art moderne de la Ville de Paris, il écrivait à propos de Réalités : «Il faudra bien un jour tenter de situer l'importance exacte de cette revue - qui est loin d'être négligeable - dans l'histoire de la photographie française d'après guerre. » Aussi, il a convaincu Bernard Boubat, le fils du photographe, de la complémentarité des deux projets. L'exposition Réalités allait mettre en avant une facette de Boubat absente de la rétrospective. Nous y reviendrons.

\section{Comment exposer une revue?}

10 L'idée de montrer la photographie imprimée et mise en page, et donc uniquement au moyen d'exemplaires de la revue, s'est imposée assez vite, confortée par la présentation de deux expositions organisées à la Maison européenne, à l'occasion du Mois de la photo 2006. La première, "Regarder $V u$ », était consacrée au premier hebdomadaire illustré d'avant-guerre. Sur deux étages, les commissaires Michel Frizot et Cédric de Veigy ont déployé quantité de couvertures et de doubles-pages témoignant non seulement d'une aventure de la presse française mais aussi d'inventions formelles et graphiques (typographie, images, textes, mise en page) au service de l'information. $V u$, qui a soutenu et valorisé la production de nombre de photographes de l'entre-deux-guerres, a servi de modèle à Life. Avec $V u$, Michel Frizot a repris un principe qu'il avait inauguré dix ans plus tôt dans l'exposition "Face à l'histoire 1933-1996, engagement, témoignage, vision » (Centre Pompidou, 19 décembre 1996 - 7 avril 1997), à savoir restituer l'impact médiatique des images en les montrant dans leur contexte de publication, c'est-à-dire reliées à un titre d'article, un texte, une légende et une mise en page. En même temps que l'exposition «Regarder $V u$ », également à la MEP, nous présentions une autre exposition mettant elle aussi en valeur la page imprimée. "L'Odyssée d'une icône» avait pour ambition de restituer l'histoire du succès de trois photographies d'André Kertész à travers leurs multiples reproductions sur différents supports, livres, magazines, journaux, cartes postales, etc.

11 Le projet de l'exposition "Réalités» était de rendre compte de l'emprise de la photographie dans la revue, de présenter ses photographes attitrés comme occasionnels, 
en se concentrant sur les deux premières décennies, les plus flamboyantes, les plus favorables à la valorisation des images. Deux directeurs artistiques incarnent cette période. Albert Gilou (1910-1961), surnommé « Bertie », occupe ce poste de 1950 jusqu'à son décès en 1961. Jacques Dumons (1936-1964), son adjoint depuis 1956, lui succède et œuvre dans la continuité de son mentor jusqu'à son décès en 1964. En 1965, la revue change de formule. Le format est réduit $(28,6$ x 21,6 cm) pour des raisons économiques. Un nouveau metteur en page, moins respectueux des photographies, est nommé. La revue devient, à notre avis, visuellement moins intéressante. Pour toutes ces raisons, l'exposition se concentrait sur les dix-huit premières années de la revue, de 1946 à 1964.

\section{Explorer les pages et l'histoire de la revue}

Pour choisir les pages à montrer et à exposer et entrer dans l'histoire de la revue, il n'y avait pas d'autre solution que d'opérer un dépouillement systématique des numéros publiés de 1946 jusqu'à la fin des années 1960. Pour les années 1970, nous avons opté pour des sondages par année. Au-delà de ce qui s'apparentait à une présélection de couvertures ou doubles-pages à exposer, il s'agissait de collecter également des informations sur les images, les sujets traités, leur ampleur, les auteurs des prises de vues ainsi que sur la revue elle-même, ses acteurs, les photographes bien entendu mais aussi les rédacteurs, les évolutions de l'équipe, du tirage de Réalités, de son prix, du nombre d'abonnés et de son audience. Ce dépouillement, effectué grâce à un tableur, prenait en compte chaque couverture ainsi que toutes les images liées à un article : le titre de chaque article illustré, les pages concernées, le nombre de photographies en noir et blanc, le nombre de photographies en couleur, le nombre de pleines pages, le nom du rédacteur, les noms des photographes et des agences mentionnés dans les crédits.

Comme Réalités était une revue d'information, il était également indispensable d'entrer dans les contenus pour comprendre la ligne éditoriale et le lectorat visé. Michel Guerrin, journaliste au Monde, spécialisé dans le photojournalisme, nous a accompagnée dans cette recherche en lisant un nombre considérable d'articles. Nous avons pu ainsi mieux évaluer les particularités de Réalités, ses orientations géopolitiques, économiques, artistiques, sa façon de traiter de l'évolution des idées et des faits de société. Nous nous sommes aussi intéressée au mode de fonctionnement de Réalités, si éloigné du rythme actuel de l'information d'aujourd'hui à l'heure d'Internet. Nous nous sommes demandé quel serait aujourd'hui l'organe de presse qui aurait pour ambition de rendre compte de l'actualité en choisissant un rythme mensuel en envoyant rédacteurs et photographes plusieurs semaines ou mois sur le terrain? Ironie de l'histoire et sans doute pour apporter une nuance à ce paysage médiatique transformé, le mensuel XXI lançait son premier numéro en même temps que notre exposition.

Pour compléter le travail de dépouillement, nous avons conduit plusieurs entretiens avec des acteurs de la revue. Nous avons d'abord rencontré Didier Rémon, un des deux fondateurs de la revue, qui nous a éclairée sur la naissance de Réalités dans le contexte de l'après-guerre et sur l'histoire du succès de ce mensuel qui reste pour lui « un conte de fée » - une formule qui balaye la fin douloureuse d'un titre qu'il a dirigé jusqu'en 1974, puis repris en 1976 sans pour autant éviter le naufrage annoncé. Il a évoqué les partenaires disparus, son ami et co-fondateur de la revue Humbert Frèrejean et le directeur de la rédaction Alfred Max. Ce dernier a laissé un petit livre de mémoires, 
publié à compte d'auteur, dans lequel nous avons trouvé quantité d'informations sur la revue $^{6}$.

Nous avons rencontré Danielle Hunebelle (1922-2013), une des premières femmes reporter et rédactrice vedette du mensuel, personnalité flamboyante et sûre de son talent, qui allait chercher l'information sur des terrains difficiles'. Elle se considérait d'ailleurs plus journaliste que ne l'étaient les photographes et ne travaillait pas avec eux de façon concertée ni sur un pied d'égalité. Elle a raconté comment elle pouvait, contrairement à Boubat, renoncer à son confort et dormir dans la rue, lors d'un reportage en Inde. Cet exemple ne doit pas laisser croire que les photographes accompagnaient régulièrement les rédacteurs. Même si nous avons identifié quelques tandems à l'occasion de plusieurs reportages, les photographes voyageaient le plus souvent seuls ${ }^{8}$. Le journaliste Gilles Anouil (né en 1933), un des principaux rédacteurs pour le mensuel, à partir de 1959, et rédacteur en chef-adjoint de 1965 à 1974, a précisé les personnalités des uns et des autres, leur relation et leur place au sein de la rédaction.

Nous avons mené parallèlement des entretiens avec des photographes salariés par Réalités , comme Michel Desjardins et Jean-Louis Swiners, ou collaborateurs réguliers comme Frank Horvat - autant de rencontres précieuses qui nous ont permis de mieux connaître leur façon de travailler et de couvrir les sujets demandés. Il manquait évidemment les deux principaux protagonistes: Boubat et Charbonnier. Ces derniers avaient cependant donné des interviews ou laissé des textes où ils évoquaient la revue ${ }^{9}$. La galeriste Agathe Gaillard aussi nous a transmis ce qu'elle savait de Réalités et de Jean-Philippe Charbonnier, auquel elle fut mariée. Ces entretiens concordent pour dire que malgré le succès de leurs images dans la revue, les photographes étaient peu considérés. Ils ne faisaient que passer dans l'immeuble de la rédaction, entre deux reportages.

\section{Les archives de Réalités}

17 Nous avons rencontré quelques membres de l'équipe qui nous ont conduite aux archives photographiques de la revue Réalités, ou plutôt ce qu'il en reste. Le succès des images publiées est à l'origine de ce fonds et de son exploitation. Avec ses photographes salariés, Réalités a en effet constitué, au fil des ans, un copieux stock de photographies. JeanPhilippe Charbonnier, notamment, produisait énormément d'images par reportage. Lui et d'autres se rendaient dans des pays peu accessibles aux voyageurs. Et quand les journaux découvraient dans Réalités des images prises en Chine, ils les achetaient pour les publier à leur tour. La revue était plutôt fière de pouvoir offrir une seconde diffusion aux images réalisées par ses photographes. En 1956, Réalités annonçait ainsi à ses lecteurs : «Il ne se passe pas un jour où quelque magazine américain, allemand, italien ou japonais ne sollicite le prêt de photographies signées Jean-Philippe Charbonnier, Édouard Boubat, Pierre-Louis Millet, Michel Desjardins ${ }^{10}$.»

18 Au fur et à mesure des reportages, un service photographique s'organise. Il est créé en 1955 et géré par Odette Fasquelle. Lily Le Roux lui succède jusqu'en 1970. Les négatifs produits, les planches-contacts correspondantes et les diapositives sont classés par sujet dans des boîtes. Les images publiées sont rangées à part dans des pochettes: toutes les images d'un même numéro dans une même pochette. Et ces pochettes sont ensuite classées dans l'ordre chronologique des numéros. Peu à peu, ce service devient véritablement une agence qui trouve son autonomie, en 1970, sous le nom de «Top », du nom d'une revue pour adolescents qui cesse de paraître cette même année. L'agence Top 
rejoint la Fédération des agences de presse. Le fonds de l'agence ne se résume pas à Réalités mais comprend également les reportages commandés pour d'autres titres du groupe. Michel Desjardins, par exemple, travaillait aussi pour Connaissance des arts. En 1975, année qui suit le départ de l'équipe dirigeante, et le démantèlement du groupe Réalités, l'agence Top est vendue. Elle est rachetée par l'agence Rapho qui enrichit ainsi son fonds d'images d'illustration.

Les archives photographiques de Réalités, que nous avons consultées en 2007, n'avaient pas fait l'objet d'un travail de valorisation. Elles comprennent une partie des images (négatifs, diapositives, planches-contacts) produites au cours des reportages, certains photographes comme Boubat et Swiners ayant récupéré leurs négatifs, ainsi qu'une collection complète de la revue. Ce fonds s'est cependant révélé très utile pour nos recherches liées à l'exposition et au livre qui l'accompagnait. Nous avons pu vérifier la production abondante de Charbonnier, le soin particulier qu'il apportait aux légendes de ses images. Sur les pochettes des diapositives, nous avons pu retrouver les noms de photographes non crédités dans la revue. Nous avons eu la bonne surprise de découvrir une pochette intitulée "maison » qui concernait la revue elle-même et rassemblait de précieux portraits de l'équipe. Ce fonds est conservé aujourd'hui au sein de l'agence Gamma-Rapho.

\section{L'exposition}

Comme nous avions décidé de présenter uniquement des exemplaires de la revue, et cela pour ne pas décontextualiser les images, il a fallu inventer un système d'accrochage, trouver un fil conducteur et rendre la présentation des couvertures et doubles-pages attractives. Nous avons d'emblée exclu l'encadrement, plutôt réservé aux œuvres, et écarté les vitrines plates, qui réduisent le nombre de revues exposables et qui sont rébarbatives et peu commodes pour un visiteur contraint de se pencher continuellement pour regarder. Bruno Bouteloup, menuisier habile et astucieux, a eu une belle idée : de grandes vitrines adossées au mur, légèrement inclinées, munies chacune de deux ou trois étagères sur toute leur longueur. Ce dispositif s'est révélé confortable pour les visiteurs que nous avons vus souvent rester un long moment à regarder les différents numéros présentés.

21 Une présentation chronologique semblait la plus à même de raconter dix-huit ans de Réalités et de traduire la place grandissante de la photographie et de la couleur. Mais avant d'entrer dans la chronologie, nous avons trouvé utile de présenter, dans une première vitrine, les deux photographes vedettes de la revue: Boubat et Charbonnier, chacun représenté par quatre doubles-pages. Une deuxième vitrine montrait cinq autres photographes de renom publiés dans Réalités : Ernst Haas, Brassaï, Cecil Beaton, Irving Penn et Henri Cartier-Bresson, chacun avec une double-page.

Pour la présentation chronologique, nous avons disposé cent quinze numéros dans trois longues vitrines qui épousaient les murs en $U$ de la salle. Une première vitrine couvrait, en quarante-trois numéros, la période «1946-1956 » et invitait le visiteur à prendre la mesure de la place grandissante de la photographie. La deuxième vitrine contenait vingtquatre numéros de la période "1956-1959», marquée par la présence de nombreuses doubles-pages en couleur. La troisième vitrine, « 1959-1964 », était consacrée à la fin de la grande période créative en une large sélection de quarante-huit numéros. 

choix de trente-trois articles. Les auteurs salariés y étaient bien représentés, en priorité Charbonnier, avec huit sujets dont « La vie ingrate et magique du médecin de campagne » (janvier 1951, $\mathrm{n}^{\circ}$ 60) ou « Bons pour l'Asile » (janvier 1955, $\mathrm{n}^{\circ}$ 108). Boubat s'imposait avec des pleines pages couleur dans les cinq articles retenus, comme : «À quoi rêvent les jeunes filles de seize ans " (octobre 1956, $\mathrm{n}^{\circ} 129$ ) ou "Grandeur et misère du paysan espagnol » (février 1958, $\mathrm{n}^{\circ} 145$ ). Desjardins était également présent à travers cinq articles dont «La guerre dans l'Aurès par ceux qui la font " (novembre 1955, $\mathrm{n}^{\circ} 118$ ). Gilles Ehrmann avait deux articles dont "Innocent, la justice me condamne" (novembre 1960, $n^{\circ}$ 178) et Frank Horvat un sujet sur la prostitution, « Un trafic qui fait honte à la France » (août 1956, n ${ }^{\circ}$ 26). La sélection montrait d'autres auteurs français comme Robert Doisneau avec « Comment camper confortablement » (septembre 1950, $\mathrm{n}^{\circ}$ 56) ou des auteurs américains comme Richard Avedon avec "Les ficelles d'une première » (décembre 1960, $\mathrm{n}^{\circ} 179$ ). Citons encore Brassaï avec « Suicide : acte conscient ou dérèglement pathologique ? (septembre 1959, $\mathrm{n}^{\circ}$ 164), ou Sergio Larrain avec un sujet sur la mafia : «La gangrène au talon de l'Europe " (avril 1960, $\mathrm{n}^{\circ} 171$ ) ou encore JeanPierre Sudre avec «Les dix plus grands restaurants de Paris» (mars 1962, $\left.\mathrm{n}^{\circ} 194\right)$. Cette sélection voulait montrer la variété des sujets abordés - la politique, les faits de société, sans oublier la culture ou les modes de vie. d'apprécier aussi l'évolution du logo. Sur la première couverture publiée, ce dernier se déploie sur toute la largeur d'une image qui n'est pas une photographie mais un dessin en couleur. Une photographie apparait pour la première fois en couverture en janvier 1949 elle est coloriée. À partir de janvier 1951, le logo est celui redessiné par Rémy Peignot - il est réduit et s'inscrit dans un bandeau de couleur. Par la suite, il ne cesse de changer de taille et d'emplacement. Il s'arrondit même pour le numéro de Noël 1955. Le logo se fait ensuite de plus en plus discret pour offrir toute la place aux images, cette dernière échappant de plus en plus au registre de l'illustration. Les auteurs des couvertures sont souvent des photographes maison. Charbonnier signe ainsi trente-six couvertures, de 1952 à 1964, et Boubat occupe vingt et une fois cette place de choix, de 1953 à 1964. Les quarante et une couvertures retenues pour l'exposition se voulaient représentatives des choix de la revue, plus de la moitié étant réalisées par des photographes salariés ou réguliers - Boubat (9), Charbonnier (7), Swiners (2), Desjardins (2), Horvat (2), Sudre (2) tout en dévoilant des images d'auteurs célèbres comme Penn (juillet 1962, $\mathrm{n}^{\circ} 198$ ) ou en devenir comme Guy Bourdin (novembre 1960, nº 178) ou Agnès Varda (avril 1962, $n^{\circ} 195$ ). Le dernier niveau mettait en valeur la qualité des images, des mises en page et des vis-àvis audacieux proposés par Albert Gilou ou Jacques Dumons. Les productions des photographes salariés ou réguliers étaient largement présentes avec, par exemple, 
Boubat : «La grande route de la brousse » (mai 1956, $\mathrm{n}^{\circ}$ 124) ou Gilles Ehrmann : «Le sort de l'Afrique noire se joue dans ses bidonvilles ", (novembre 1959, $\mathrm{n}^{\circ} 166$ ) mais aussi des photographes de réputation internationale comme Elliott Erwitt: «Pour une politique française au Moyen Orient ", (février 1959, $n^{\circ}$ 157), Manuel Alvarez Bravo : «Quand Dieu cohabite avec les idoles » (janvier 1960, $\left.\mathrm{n}^{\circ} 168\right)$.

Une autre difficulté a été de trouver des exemplaires de la revue que l'on pouvait ouvrir aux pages sélectionnées. Il était par exemple impossible de les emprunter à une bibliothèque qui les conserve généralement reliés. L'exposition a donc pu se construire grâce aux collections de quelques particuliers et grâce à celle de la MEP. L'agence Rapho a pour sa part prêté quelques numéros, notamment les plus anciens. Certains numéros offraient plusieurs pages à exposer et il a donc fallu en trouver plusieurs exemplaires deux ${ }^{11}$, trois ${ }^{12}$, voire quatre (mai 1959, $\mathrm{n}^{\circ} 60$ ).

L'exposition Réalités racontait à la fois une époque, la présence soutenue de la photographie dans une revue et le métier de reporter-photographe dans les années 1950-1960. Les photographes attachés à Réalités aimaient surtout opérer en noir et blanc mais la direction leur imposait aussi la couleur. Ils partaient donc avec deux appareils pour doubler les prises de vue. Certains comme Desjardins avaient même quatre appareils, deux $24 \times 36$ et deux moyens formats. Exposer la revue a donc permis de découvrir des images en couleur que certains photographes comme Boubat ou Charbonnier n'ont jamais tirées, préférant en montrer la version en noir et blanc dans des expositions ou sur les cimaises des galeries, quand ils se sont lancés dans la vente de tirages, à partir de 1975.

Sans les publications dans Réalités, nous ne saurions pas aujourd'hui à quel point cette génération a pratiqué la couleur. Et souvent avec talent. Mais la plupart des photographes considéraient le procédé couleur comme banal, réservé à la publicité, inadapté à l'instantané ou offrant des effets faciles. Pour les photographes de Réalités, l'expérience vécue après 1965, avec une revue toute en couleurs et des images recadrées à tout-va, a certainement favorisé le rejet en bloc de cette production. Réalités nous montre cependant de nombreuses variantes en couleur d'images connues de Boubat souvent publiées en couverture comme la petite fille de Grenade, " À Grenade où les enfants sont comme des Velasquez " (septembre 1955, $\mathrm{n}^{\circ} 116$ ), ou la maternité de "Bethléem» (décembre 1954, $\mathrm{n}^{\circ}$ 107), mais aussi dans les pages du magazine, comme les enfants noirs derrière une vitre, qui illustrent dans un dossier spécial sur L'Amérique vue par les Français un article sur « La question noire » (août 1953, n 91). D'autres images, comme ce visage de femme en couverture d' «Être noir en Alabama " (avril 1964, $n^{\circ}$ 219) ou cette rue animée, vue depuis l'intérieur d'un restaurant à Delft (Pays-Bas), publiée en double-page dans l'article « La nature profonde de l'Europe » (juin 1959, n 161) échappent à l'image de poète intemporel que Boubat a voulu laisser. Toutes ces images en couleur ancrées dans la réalité d'une époque posent aujourd'hui plusieurs questions: quelle place leur donner dans l'œuvre du photographe et comment les montrer? Choisir les images publiées est une façon de les rendre visibles sans pour autant trahir le photographe qui n'avait pas choisi d'en réaliser des tirages. 


\section{NOTES}

1. - Voir le site : https://www.mep-fr.org/event/realites/ [consulté le 21/08/2018].

2. - Voir MONDENARD, Anne de et GUERRIN, Michel. Réalités. Un mensuel français illustré. Arles : Actes Sud, 2008.

3. - MAX, Alfred. 30 ans d'initiatives et d'affrontements en lisière de l'Histoire : sondages, presse, politique locale. Plan-de-la-Tour : éd. d'Aujourd'hui, 1988, p. 51-52.

4. - Voir TIMBY, Kim. "La photographie en couleurs au prisme de la presse française, 1945-1960 ». Focales, $n^{\circ} 1$ : Le photographe face au flux, mis à jour le 25/08/2017, URL : http://focales.univ-st-etienne.fr/index.php?id=507. Dans cet article, Kim Timby compare la place de la couleur dans Paris-Match et Réalités: «en moyenne, Réalités publie 1,2 photographies en couleurs par numéro en 1946 ; puis 2,3 en $1947 ; 10,3$ en $1948 ; 11,8$ en 1949 et 15,2 en 1950 . [...] Les photographies en couleurs se multiplient de la création du magazine jusqu'en 1953, atteignant cette année-là une moyenne de 19,7 par mois; puis leur nombre décroît jusqu'à la fin de la décennie, avec une moyenne de 9,9 par mois en 1959 [...] ; elles sont plus grandes et très souvent en pleine page. » Nous regrettons qu'elle ait cité de façon tronquée une phrase de notre ouvrage pour nous faire dire des généralités sur la presse illustrée alors que nous évoquions simplement le cas de Réalités (op. cit., p. 51).

5. - Pour un historique de la revue et des visuels des autres titres, voir http://docpresse.esjlille.fr/realites/ [consulté le 21/08/2018].

6. - MAX, Alfred. Op. cit.

7. - Voir RIMBAUD, Christiane. Danielle Hunebelle grand reporter. Paris : A. Carrière, 2001.

8. - Charbonnier et Philippe Soupault à Brazzaville, en 1951; Boubat et Georges Rotvand en Algérie, en 1954; Boubat et Danielle Hunebelle, en Afrique en 1956 et en Inde, en 1962; Desjardins et Pierre Marchant en Colombie, en 1962 ; Swiners et Michel Del Castillo au Mexique, en 1962 ; Swiners et Muriel Reed (accompagnée de Soupault) en Albanie, en 1964 ; Agnès Varda et Gilles Anouil en Bavière, en 1961.

9. - TALAMON, Sophie. «Entretien avec Jean-Philippe Charbonnier ». Photographie nouvelle, $\mathrm{n}^{\circ} 27$, Noël/janvier 1967 ; Jean-Philippe Charbonnier. Photographies. Paris : musée d'Art moderne de la Ville de Paris/Paris-Musées, 1983. BOUBAT, Édouard. Préférées. Paris: Contrejour, 1980; BOUBAT, Édouard. Pauses. Paris : Contrejour, 1983.

10. - «Quatorze inédits de nos photographes ». Réalités, $\mathrm{n}^{\circ} 122, \mathrm{p} .92$.

11. - Les numéros 107, 118, 121, 124, 130, 131, 144, 145, 147, 158, 161, 162, 171, 174, 178, 179, 182, 191, 194, 195, 198, 202, 206, 219.

12. - Les numéros $122,156,157,168,220$.

\section{RÉSUMÉS}

Le propos de cet article est de retracer les enjeux qui ont présidé à la présentation de l'exposition «Réalités un mensuel illustré français (1946-1978) » à la Maison européenne de la photographie, 
en 2008. Créé en 1946, Réalités a pour ambition de raconter l'actualité sur un rythme mensuel. Il a cessé de paraître en 1978. Longtemps oublié, il est pourtant un des titres les plus novateurs et les plus influents des années 1950-1960. Son audience le place alors parmi les journaux les plus lus et les plus regardés en France. Le mensuel a donné une place grandissante à la photographie en recrutant des photographes comme Édouard Boubat et Jean-Philippe Charbonnier, tout en publiant les auteurs les plus actifs et talentueux de la période : Haas, Penn, Avedon, etc. Le projet de l'exposition était de rendre compte de la place prise par la photographie dans la revue, pendant les années les plus flamboyantes (1946-1964). Deux directeurs artistiques incarnent cette période : Albert Gilou (1950-1961) et son adjoint Jacques Dumons (1961-1964). En 1965, la revue change de format pour des raisons économiques. Elle est visuellement moins intéressante. L'exposition ne proposait pas des tirages des images publiées mais des photographies imprimées et mises en page, pour attirer l'attention sur des couvertures, des articles illustrés ou des doubles pages, selon une organisation et un dispositif attractifs pour le visiteur.

The ambition of this article is to take a retrospective look at the exhibition 'Réalités, an illustrated French monthly (1946-1978)', put on at the Maison européenne de la photographie in Paris in 2008. Réalités, founded in 1946, was a monthly news magazine which ceased publication in 1978. Largely forgotten today, it was nonetheless one of the most innovatory and influential reviews of the 1950s and 1960s with circulation figures which placed it amongst the magazines which were the most read, and looked at, in France. The magazine gave ever-increasing importance to the place of photography, recruiting photographers like Édouard Boubat and Jean-Philippe Charbonnier and publishing the work of other talented photographers such as Haas, Penn or Avedon. The aim of the exhibition was to show the importance of photography in the magazine during its heyday, from 1946 to 1964. Two artistic directors were important during these years, Albert Gilou (1950-1961) and his assistant Jacques Dumons (1961-1964). In 1965, for economic reasons, the magazine changed its format and, visually, became less interesting. The exhibition did not show prints of the photos that were published in the magazine, but the photos as they were actually printed and placed in the layout of the magazine's pages, drawing attention to covers, to illustrated articles or to double-page photo spreads. The exhibition was organised and presented in as attractive a way as possible.

\section{INDEX}

Mots-clés : photographie, Réalités, Presse illustrée, Trente Glorieuses, Mensuel, Alfred Max, Albert Gilou, Jacques Dumons, Édouard Boubat, Jean-Philippe Charbonnier, Exposition, Maison européenne de la photographie, Mise en page

Keywords : photography, Réalités, illustrated press, 'Thirty Glorious Years', monthly, magazine, Alfred Max, Albert Gilou, Jacques Dumons, Édouard Boubat, Jean-Philippe Charbonnier, Exposition, Maison européenne de la photographie, layout

\section{AUTEUR}

\section{ANNE DE MONDENARD}

Docteur en histoire de l'art, conservateur en chef du patrimoine, responsable du département Photographies et Images numériques, musée Carnavalet - Histoire de Paris Anne.DeMondenard@paris.fr 